\title{
Belgeo
}

Revue belge de géographie

3-4 | 2008

Formatting Europe - Mapping a Continent

\section{Introduction : Cartes d'Europe : quelques réflexions}

\section{Wouter Bracke}

\section{OpenEdition}

\section{Journals}

Édition électronique

URL : http://journals.openedition.org/belgeo/7655

DOI : $10.4000 /$ belgeo.7655

ISSN : 2294-9135

\section{Éditeur :}

National Committee of Geography of Belgium, Société Royale Belge de Géographie

\section{Édition imprimée}

Date de publication : 31 décembre 2008

Pagination : 245-250

ISSN : 1377-2368

\section{Référence électronique}

Wouter Bracke, «Introduction: Cartes d'Europe : quelques réflexions », Belgeo [En ligne], 3-4 | 2008,

mis en ligne le 22 mai 2013, consulté le 05 février 2021. URL : http://journals.openedition.org/belgeo/ 7655 ; DOI : https://doi.org/10.4000/belgeo.7655

Ce document a été généré automatiquement le 5 février 2021.

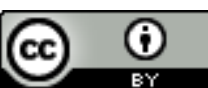

Belgeo est mis à disposition selon les termes de la licence Creative Commons Attribution 4.0 International. 


\title{
Introduction : Cartes d'Europe : quelques réflexions
}

\author{
Wouter Bracke
}

1 Les cartes constituent, à l'instar des livres, un moyen de communication: elles racontent une histoire. De la même façon qu'il existe de nombreuses sortes d'histoires et donc de livres, nous disposons d'une panoplie de cartes différentes. Tout ce qui peut être conçu de manière spatiale peut faire l'objet d'une carte. La forme de la carte dépend du cartographe (auteur, graveur ou éditeur), elle est déterminée par ses connaissances et ses capacités techniques ou artistiques, par le message qu'il veut transmettre et le groupe cible auquel il s'adresse.

2 Pendant longtemps, la précision scientifique n'a pas été une préoccupation majeure des cartographes. Aujourd'hui encore, elle n'est qu'une motivation parmi d'autres dans la réalisation d'une carte. Ainsi les cartes thématiques (politiques, idéologiques, économiques, touristiques...) vont-elles privilégier le message au détriment d'une reproduction fidèle de la réalité géographique. Les cartes de métro en sont, sans conteste, l'exemple le plus frappant et le plus extrême. Mais même à l'intérieur d'un même genre les cartes peuvent être très différentes les unes des autres. Pour rester dans les sujets traités ici, la carte hypsométrique de Jean-Charles Houzeau est très différente de celle de Berghaus ou de celle d'André Dumont. Elles sont pourtant toutes $\mathrm{du} \mathrm{XIX}^{\mathrm{e}}$ siècle. Savoirs technique et artistique, traditions cartographiques dans lesquels les auteurs sont formés, semblent avoir joué un rôle décisif. Ainsi, la carte de Houzeau ne plut guère au milieu cartographique allemand de l'époque. Mais aussi avant le XIX ${ }^{e}$ siècle, période de grands changements techniques, les possibilités de la technique jouaient un rôle important. La xylographie n'offre pas les mêmes possibilités que la gravure sur cuivre. Cette dernière par contre fournissait un produit similaire à la lithographie. Le choix d'une de ces techniques avait des conséquences majeures pour la production cartographique. La gravure sur cuivre est une technique coûteuse et lente. Pour couvrir les frais de la production, il fallait déjà vendre bon nombre de cartes. Les tirages étaient donc importants et les plaques devaient servir le plus longtemps possible. Certaines plaques étaient utilisées par plusieurs générations de cartographes 
ou furent vendues d'un cartographe à l'autre. Par conséquent, certaines cartes montrent, malgré les petites corrections qu'on y intégrait de temps en temps, un savoir géographique, une réalité politique ou des tendances stylistiques tout à fait dépassés par rapport à l'époque de leur impression.

La lecture d'un livre requiert une certaine formation. Outre sa capacité de lecture et sa connaissance de la langue dans laquelle est écrit l'ouvrage, le lecteur doit aussi se familiariser avec le langage propre à l'auteur et, de préférence, avoir quelques connaissances rudimentaires du sujet traité.

4 Au même titre, les cartes requièrent une certaine formation. Outre le fait qu'elles font appel à un langage spécifique, il est souhaitable que leur utilisateur ait quelques notions sur la période où elles ont été créées, sur leur raison d'être, leur auteur, graveur ou éditeur. Or, c'est là que commence le problème car si certains cartographes sont très connus, pour d'autres nous ne connaissons que le nom. De même, le langage utilisé par les cartographes n'est pas toujours univoque et peut donner lieu à des interprétations divergentes, voire des malentendus. En outre, l'iconographie employée n'est pas toujours comprise de la même façon par tous et un même symbole repris sur des cartes différentes ne renvoie pas toujours au même sujet.

5 En offrant des possibilités d'interprétation multiples, les cartes font souvent l'objet d'interprétations différentes au fil du temps, selon le contexte historique ou social. Elles peuvent tout aussi bien être une représentation correcte de la réalité géographique que l'expression d'une imagination débordante. Tantôt véridiques, mais tantôt occultant volontairement la vérité, elles n'ont pas peur du mensonge !

Les cartes constituent donc des documents historiques précieux comme témoins de l'évolution de l'homme. Ce sont des produits de leur époque qui tentent de répondre aux exigences de la société dans laquelle elles voient le jour, aux objectifs du commanditaire, et à la demande du public. Elles illustrent ainsi la mentalité d'une société déterminée à un moment précis et s'adaptent aux circonstances nouvelles et à l'évolution des mentalités changeantes. Les cartes sont porteuses de changement.

7 L'exposition que nous avons organisée en 2007-2008 dans le cadre d'Europalia-Europe, à l'occasion de laquelle le colloque dont nous publions ici les actes fut organisé, couvrait dix siècles de cartographie. Le document le plus ancien remontait en effet au $\mathrm{XI}^{\mathrm{e}}$ siècle. Il s'agissait d'un manuscrit de Salluste provenant de l'abbaye d'Egmond aux Pays-Bas. Mais si le document était du $\mathrm{XI}^{\mathrm{e}}$ siècle, l'image de l'Europe reproduite dans ce manuscrit est encore plus ancienne puisqu'elle reflète la vision du monde de l'auteur telle qu'il l'a décrite au premier siècle avant notre ère. L'exposition donnait ainsi une idée de la façon dont notre vieux continent était représenté, ou pour le moins imaginé, durant l'Antiquité déjà.

Les premiers documents que nous montrions, qui n'étaient pas des cartes, eurent trait à l'historien Hérodote, au poète bucolique Moschos et à son collègue Ovide. Les oeuvres de ces derniers sont à la base de la diffusion du fameux mythe de l'enlèvement d'Europe par le dieu Zeus, mais aussi d'une première tradition figurative dans la représentation $\mathrm{du}$ vieux continent. Cette vision globale/mondiale fait apparaître un continent tributaire de l'Asie, qui n'occupe finalement qu'une partie modeste du monde connu.

9 Notre choix des documents cartographiques avait été d'emblée orienté par deux questions : il s'agissait de s'interroger d'une part sur l'évolution de la représentation européenne dans les cartes; de l'autre, sur le témoignage que livrent ces cartes qui 
traitent toutes un sujet identique et qui sont souvent de forme semblable, voire similaire. Pour répondre à ces questions, nous avions regroupé les cartes en cinq périodes chronologiques soulignant les étapes importantes de leur histoire cartographique.

Avant tout, l'évolution se manifeste évidemment dans le progrès scientifique : le calcul et l'utilisation des longitudes et latitudes avec notamment le problème de la mer Méditerranée, l'établissement de la frontière orientale de l'Europe ; la lente découverte du nord de l'Europe; le méridien d'origine. Vient ensuite le style des cartes. Une des salles proposait en effet dans la tradition bien connue d'Ortelius et de Mercator une série de cartes d'atlas, qui couvraient les $\mathrm{XVII}^{\mathrm{e}}$ et $\mathrm{XVIII}{ }^{\mathrm{e}}$ siècles. On pouvait ainsi découvrir au premier coup d'oeil les divergences d'un siècle à l'autre, notamment au niveau de la décoration, des cartouches, du coloriage, et ce malgré une unité évidente et indéniable. Cette unité dans la production cartographique, on la cherchait en vain dans les cartes que nous avions choisies pour les $\mathrm{XIX}^{\mathrm{e}}$ et $\mathrm{XX}^{\mathrm{e}}$ siècles. A cette période, on assiste en effet à une plus grande diversification de la production qui va de pair avec une plus grande individualisation. Tout sentiment d'école ou d'atelier cartographique semble très loin. La carte n'est que rarement un but en soi. Elle est secondaire, fonctionnelle et auxiliaire. On assiste à l'essor de la carte thématique. Parfois, des constatations étonnantes s'imposaient: par exemple, la réapparition des décorations marginales, si caractéristiques des cartes de Hondius et de Blaeu, dans la carte d'Aristide-Michel Perrot en 1841, avec ses plans de villes et ses couples en costumes traditionnels (voir figure).

11 L'histoire s'arrêtait dans les années 1950 au moment du traité de Rome. Ce traité signifia la naissance de l'unification européenne et en même temps d'une nouvelle génération de cartes générales de l'Europe ou mieux de l'Union européenne.

Même si l'exposition souhaitait montrer l'évolution de la représentation cartographique du vieux continent sans parti pris politique et sans vouloir défendre d'idéologie politique, il va sans dire que l'approche géopolitique de la cartographie de l'Europe occupe une place non négligeable dans ce domaine. Les cartes peuvent en effet jouer un rôle politique et ce fut souvent le cas dans le passé. Les souverains et autres princes régnants voulaient dresser la carte des régions qui étaient sous leur domination. Les guerres ont engendré des déplacements internes et externes de frontières. De grands traités de paix internationaux ont redessiné le vieux continent. Les cartographes répondaient à ces évolutions: ils montraient des (propositions de) lignes de combat ; faisaient le compte rendu des batailles livrées ; éditaient des cartes détaillées avec des informations militaires importantes sur les gués des rivières et des fleuves, le boisement et la construction, le relief...; montraient de nouvelles entités nationales ou régionales et critiquaient au passage, le cas échéant, les rapports de force existants.

La politique n'est toutefois qu'un motif parmi d'autres des cartes qui faisaient l'objet de cette exposition. L'une des conclusions que l'on pourrait tirer de cette exposition serait d'ailleurs que certaines traditions cartographiques ont souvent été plus déterminantes que la géopolitique. En effet, même si les cartes présentent une grande diversité de formes, elles ont ceci en commun que les cartographes représentaient un ensemble géographique tel qu'ils le concevaient eux-mêmes dans le cadre de traditions cartographiques bien précises. 
Europa recens descripta a Guilielmo Blaeuw, [Amsterdam, après 1660], CP XII - Europe gén. - XVII s. - III 10 920. Copyright Bibliothèque royale de Belgique.

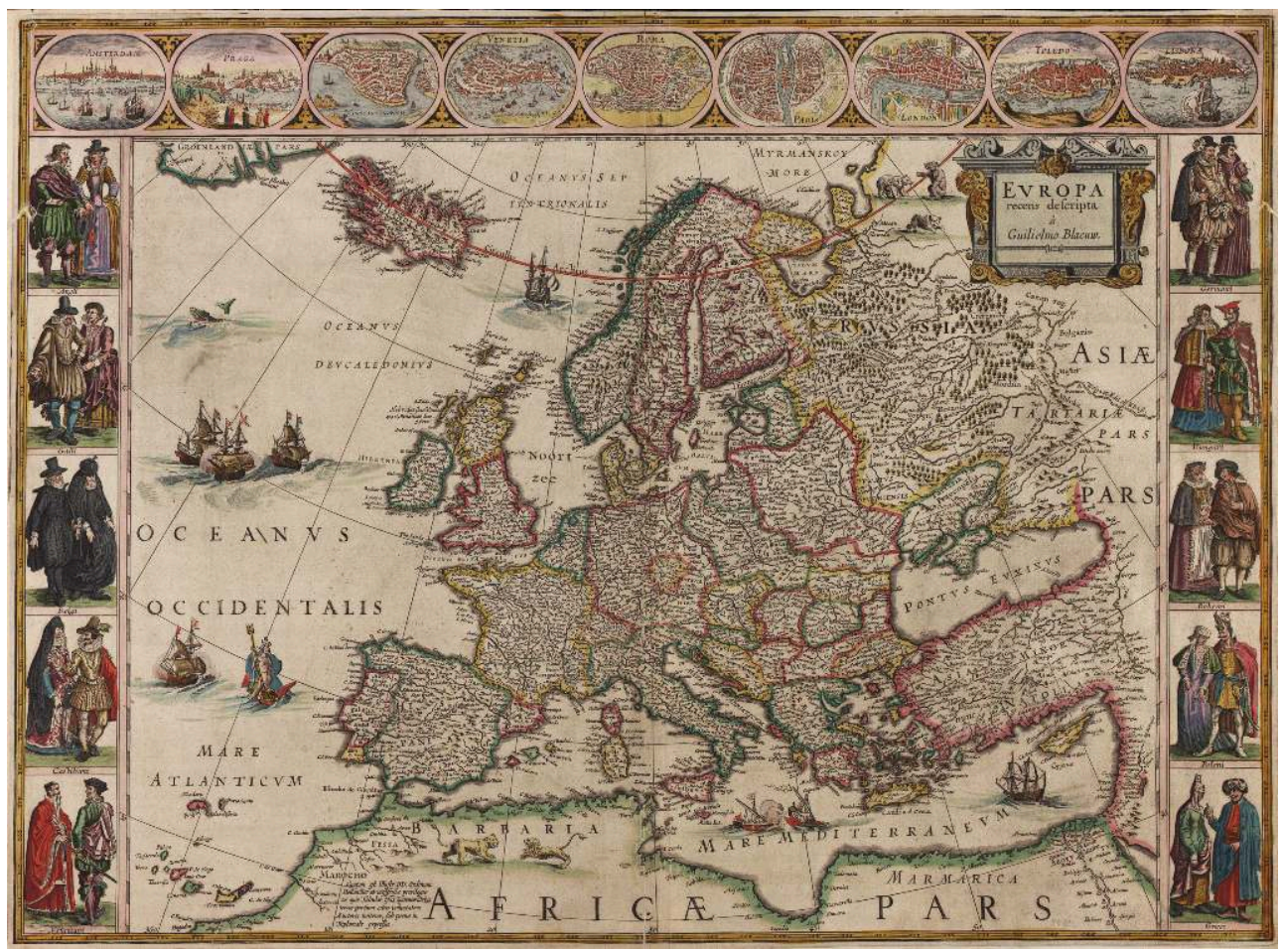

Carte pittoresque et maritime de l'Europe indiquant la division des Etats, les routes et chemins de fer, la distance en kilomètres de Paris à toutes les villes importantes, les Productions [...], par A.M. Perrot, Paris, [1841 ?], CP XII - Europe gén. - 1841 - Perrot - III 8 596. Copyright Bibliothèque royale de Belgique.

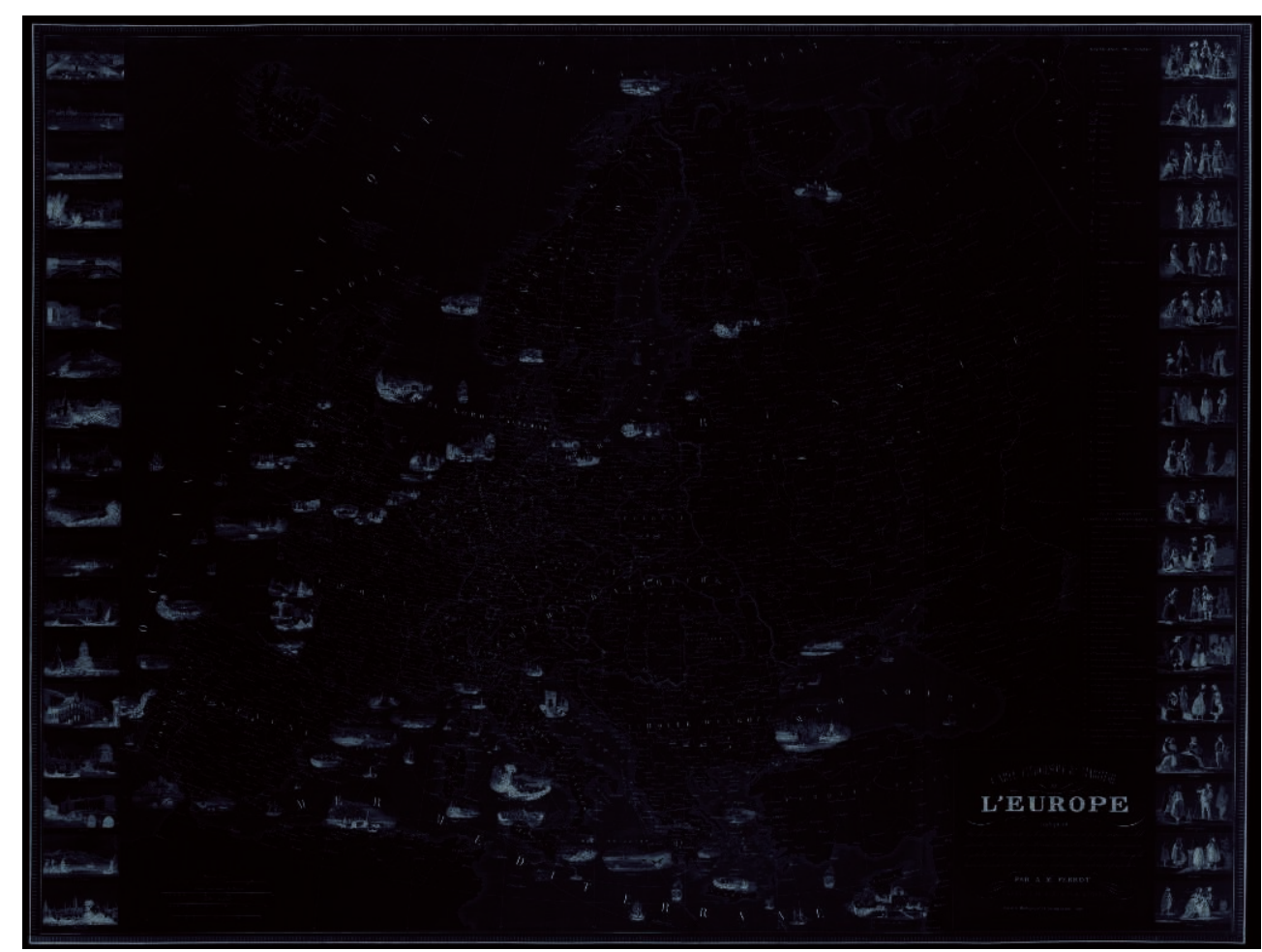




\section{AUTEUR}

WOUTER BRACKE

Bibliothèque royale de Belgique, wouter.bracke@kbr.be 